\title{
EXPRESSION OF CYTOSKELETAL PROTEINS IN HEPATIC STELLATE CELLS ISOLATED FROM NORMAL AND CIRRHOTIC RAT LIVER
}

\author{
Alena Jiroutová, Lenka Majdiaková, Martina Čermáková, Renata Köhlerová, Jiří Kanta \\ Charles University in Prague, Faculty of Medicine in Hradec Králové, Czech Republic: Department of Medical \\ Biochemistry

\begin{abstract}
Summary: Hepatic stellate cells (HSC) are located in Disse spaces of normal rat liver. In their quiescent state they serve as a storage site for vitamin A. In fibrotic liver they become activated, proliferate and they undergo transdifferentiation into myofibroblast-like cells. Changes in the cell phenotype are accompanied by changes in the cellular cytoskeleton. We have studied the expression of $\alpha$-smooth muscle actin and intermediate filament proteins vimentin, desmin and glial fibrillary acidic protein (GFAP) by immunocytochemistry in HSC cultured for 2 or 7 days after isolation. Normal or cirrhotic rat liver was perfused with solutions of pronase and collagenase and HSC were isolated by density gradient centrifugation of the resulting cell suspension. Liver cirrhosis was produced in rats by repeated carbon tetrachloride administration. Vimentin was detected in all cells from normal and cirrhotic liver. The concentration of desmin in the cells from cirrhotic liver was slightly higher than that in normal cells and it increased with time in culture. GFAP could be detected only in normal cells 2 days after their isolation. In contrast, alpha smooth muscle actin ( $\alpha$-SMA) was absent from normal cells at this time but its expression was pronouced later. In most cells from cirrhotic liver this antigen was already present on the second day of culture and its expression further increased.
\end{abstract}

Key words: Liver cirrhosis; Carbon tetrachloride; Hepatic stellate cells; Vimentin; Desmin; Glial fibrillary acidic protein; $\alpha$-smooth muscle actin

\section{Introduction}

Hepatic stellate cells (HSC) are localized in the spaces of Disse and their long cytoplasmic processes are in close contact with neighbouring cells, sinusoidal endothelial cells and hepatocytes. In normal liver, an important function of HSC is storing vitamin A, mainly in the form of retinyl esters. HSC synthesize various types of extracellular matrix (ECM) components, fibrillar collagen types I, III and V, net-forming collagen type IV, glycoproteins laminin and fibronectin, hyaluronic acid and proteoglycans containing heparan, dermatan and chondroitin sulfate. The composition of normal liver ECM resembles that of basement membranes but no continuous membrane is present. Together with the fenestrations of endothelial cells it allows the exchange of metabolites between hepatocytes and blood. Some ECM proteins are synthesized by liver endothelial cells $(10,16,19)$.

In the process of fibrogenesis, HSC are stimulated to proliferate and to undergo phenotypic transformation to contractile cells that are thought to produce majority of connective tissue in diseased liver. In damaged liver, HSC secrete a number of cytokines and growth factors, while their ability to degrade ECM is decreased. This process is called activation or transdifferentiation and is accompanied by changes in the cellular cytoskeleton. HSC cultured on plastic for 2 days are regarded as quiescent. The quiescent phenotype can be maintained when the cells are cultured on basement membrane-like substrate. When they are cultured on plastic substrate for 5 to 7 days, they acquire activated, myofibroblast-like phenotype $(4,8,16)$. They synthesize and secrete increasing amounts of ECM proteins and they are used to study the processes leading to ECM deposition in liver fibrosis $(4,6)$.

Stress fibres containing $\alpha$-smooth muscle actin $(\alpha-$ SMA) are a characteristic feature of activated HSC (17). Intermediate filaments also contain proteins that can be used as markers for HSC. Isolated HSC are positive for vimentin when cultured for 3 or 7 days on plastic $(2,17)$. Desmin can be detected both 3 and 7 days after isolation and its expression in HSC increases during cultivation (7). Expression of glial fibrillary acidic protein (GFAP) is high after isolation but decreases quickly in culture (12).

The properties of HSC isolated from normal liver and activated by culture on plastic substrate may be different from the properties of HSC present in cirrhotic rat liver. However, the characterization of the four cytoskeletal antigens in the cells from cirrhotic liver in the literature is in- 
complete and the way of fibrosis and cirrhosis induction as well as the length of treatment vary. We have compared the expression of these antigens in normal HSC and in HSC isolated from cirrhotic liver. Cirrhosis was induced in rats by repeated application of carbon tetrachloride and the expression of the four markers, $\alpha$-SMA, vimentin, desmin and GFAP was determined by immunocytochemistry. The cells were examined at two time intervals, 2 days after isolation when they attached to the substrate and at 7 days when they were spread and reached confluency.

\section{Materials and Methods}

\section{Chemicals}

Carbon tetrachloride was obtained from Riedel-de Haen (Seelze, Germany), collagenase B, pronase and DNase I from Roche (Mannheim, Germany), Hank's balanced salt solution (HBSS) from Gibco (Karslruhe, Germany), Optiprep from Nycomed Pharma (Oslo, Norway), bovine serum albumin and Oil Red $\mathrm{O}$ from Fluka (Buchs, Switzerland). Fetal bovine serum was obtained from PAA Laboratories (Cölbe, Germany). Dulbecco medium, fish gelatin and monoclonal antibodies against GFAP (clone G-A-5) and $\alpha$-SMA (clone 1A4) were from Sigma-Aldrich (Prague, Czech Republic). Monoclonal antibodies against vimentin (clone V9) and desmin (clone DER-11) and secondary antibody coupled to horseradish peroxidase and diaminobenzidine were obtained from Dako (Glostrup, Denmark), mouse gamma globulins from Jackson ImmunoResearch (West Grove, USA). Other chemicals were from Serva (Heidelberg, Germany).

\section{Animal treatment, cell isolation and culture}

Male Wistar rats weighing 250-300 g were obtained from VUFB breeding station (Konárovice, Czech Republic). The rats received humane treatment in accordance with Czech laws. Cirrhosis was produced by giving the rats $1 \mathrm{ml} / \mathrm{kg} \mathrm{CCl}_{4}$ by gavage twice a week for 9 weeks. The toxin was disolved in olive oil 1:1 (v/v).

Hepatic stellate cells were isolated from normal liver or from cirrhotic liver 3 days after the last $\mathrm{CCl}_{4}$ dose and the procedure described by Knook et al. (9) was adapted. The liver was perfused through the portal vein in situ successively with $\mathrm{Ca}^{2+}$-free $\mathrm{HBSS}$ for $10 \mathrm{~min}$., with $100 \mathrm{ml} 0.2 \%$ pronase solution and with $0.013 \%$ collagenase solution. The enzymes were dissolved in complete HBSS and the rate of perfusion was $10 \mathrm{ml} / \mathrm{min}$. It was stopped when the liver looked digested. The resulting cell suspension was incubated with $0.01 \%$ DNase for $15 \mathrm{~min}$. at $37{ }^{\circ} \mathrm{C}$ in a shaking water bath and then filtered through $42 \mu$ m nylon mesh (Silk \& Progress, Moravská Chrastová, Czech Republic). The cells were centrifuged at $400 \mathrm{~g}$ for $7 \mathrm{~min}$. and washed with HBSS. Commercially available $60 \%$ Optiprep was added to the final concentration $11.5 \%$ (3) and the suspension overlaid with $0.5 \mathrm{ml} \mathrm{HBSS}$ was centrifuged at $1400 \mathrm{~g}$ for $22 \mathrm{~min}$. The cells on the top of Optiprep layer were collected.
The cells were cultured in Dulbecco medium containing $10 \%$ fetal calf serum, $4 \mathrm{mM}$ L-glutamine, $100 \mathrm{U} / \mathrm{ml}$ penicillin and $100 \mu \mathrm{g} / \mathrm{ml}$ streptomycin. They were harvested 2 and 7 days after plating, respectively. They were fixed in $4 \%$ paraformaldehyde dissolved in phosphate-buffered saline (PBS) and kept in PBS at $4{ }^{\circ} \mathrm{C}$ until use.

A portion of the right lateral liver lobe removed at the beginning of the perfusion was fixed in $4 \%$ formaldehyde, embedded in paraffin and saved for histological examination.

\section{Oil Red staining, immunocytochemistry and immunohistochemistry}

The cells fixed in paraformaldehyde were incubated with saturated Oil Red $\mathrm{O}$ solution in $70 \%$ ethanol for 15 min. and washed with $50 \%$ ethanol and water.

For immunocytochemistry, the cells were permeabilized on ice by cold solution of $0.1 \%$ Triton X-100 in $0.1 \%$ sodium citrate for $2 \mathrm{~min}$. Endogeneous peroxidase was blocked by $3 \%$ hydrogen peroxide for $30 \mathrm{~min}$. Nonspecific binding sites were blocked by $50 \%$ fetal calf serum in PBS and by a solution of $0.1 \%$ fish gelatin and $0.5 \%$ bovine serum albumin in PBS. The cells were incubated with the primary antibody at room temperature for 2 hours. Anti -vimentin antibody was diluted 1:100, anti-desmin 1:50, antiGFAP 1:400 and anti- $\alpha$-SMA 1:400. The kit containing the secondary antibody and peroxidase was used according to the instructions of the manufacturer.

Histological sections of liver tissue were deparaffinized and stained with haematoxylin-eosin, van Gieson stain and by silver impregnation according to Gömöri.

\section{Results}

The livers of rats treated with $\mathrm{CCl}_{4}$ for 9 weeks had nodular appearance and some of the rats had ascites. When examined under microscope, liver architecture was distorted with broad connective tissue septa separating regenerative nodules. Hepatocyte degeneration was seen. Argyrophilic material was found in the sinusoids of normal liver but in cirrhotic liver mainly the septa gave a positive reaction (Fig. 1 a-f).

Hepatic stellate cells from both normal and cirrhotic livers contained fat droplets stained with Oil Red O. They were present in both cell types 2 days after isolation and persisted for 7 days of cultivation (Fig. 2 a-d).

HSC from normal liver did not contain $\alpha$-SMA 2 days after isolation but about one half of them were positive when they were cultured for 5 more days. Eighty percent of HSC from cirrhotic liver expressed $\alpha$-SMA as early as 2 days after isolation and almost 100\% cells showed strong expression of $\alpha$-SMA at 7 days. The cells also increased in size (Fig. 3 a-d).

Vimentin was detected in both cell types at the two time intervals examined. It was present in all HSC and its expression increased during cultivation (Fig. 4 a-d). Desmin 


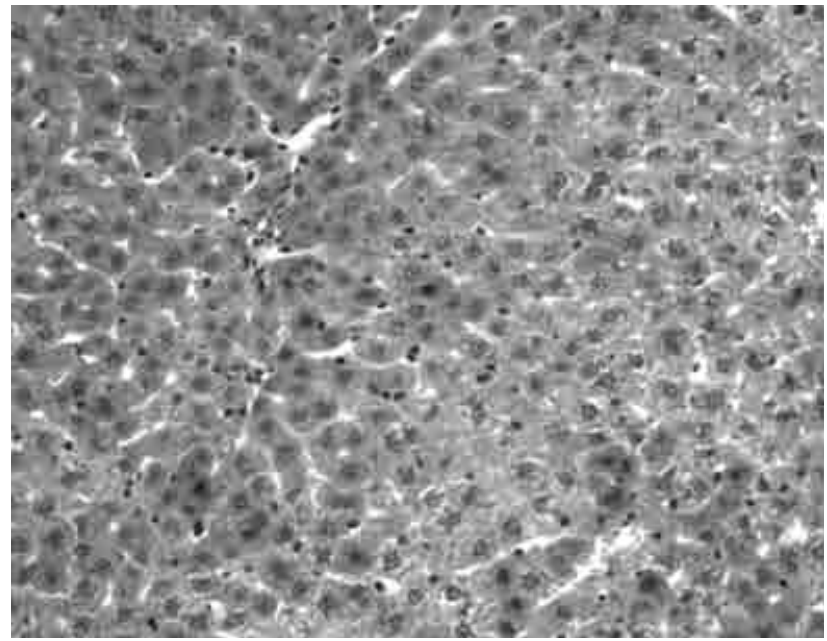

Fig. 1a: Normal liver, haematoxylin-eosin.

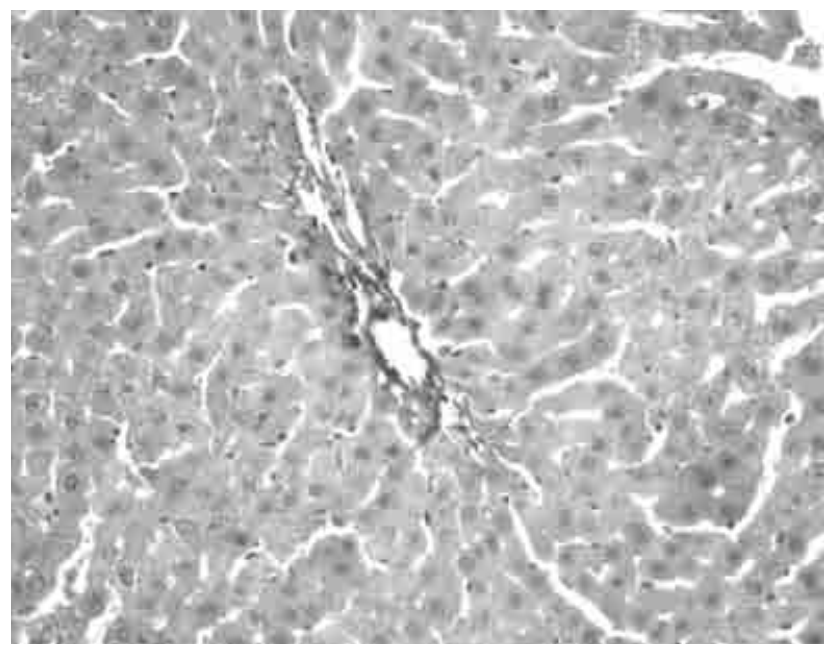

Fig. 1c: Normal liver, van Gieson technique.

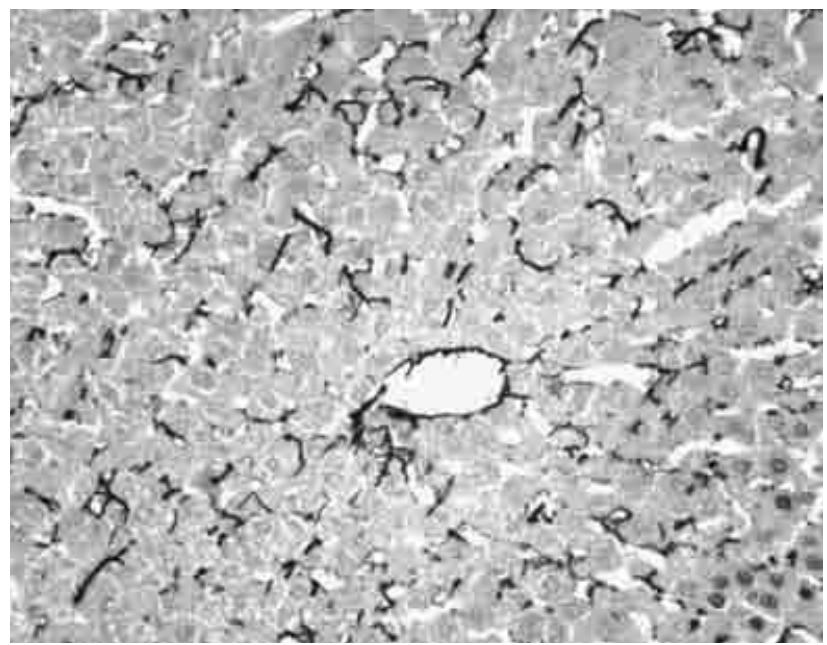

Fig. 1e: Normal liver, Gömöri‘s silver impregnation.

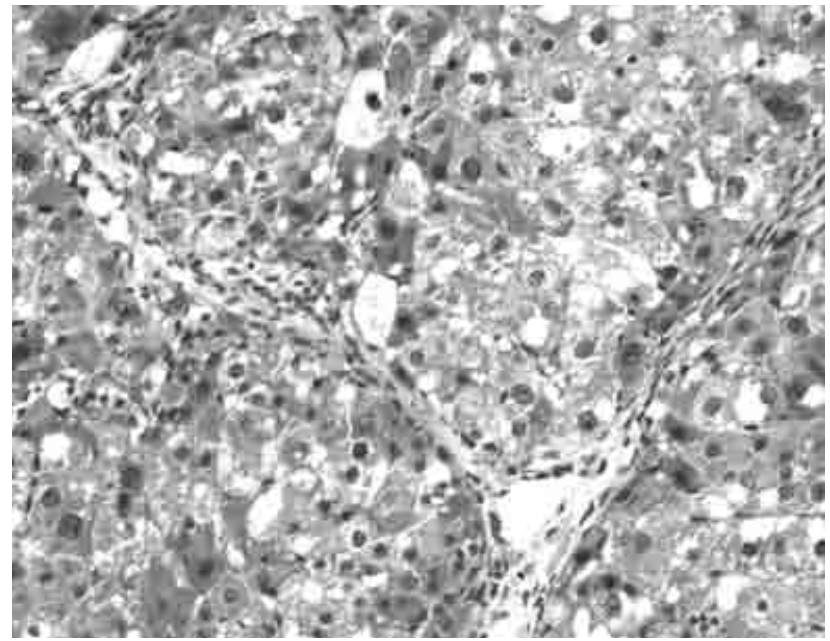

Fig. 1b: Cirrhotic liver, haematoxylin-eosin.

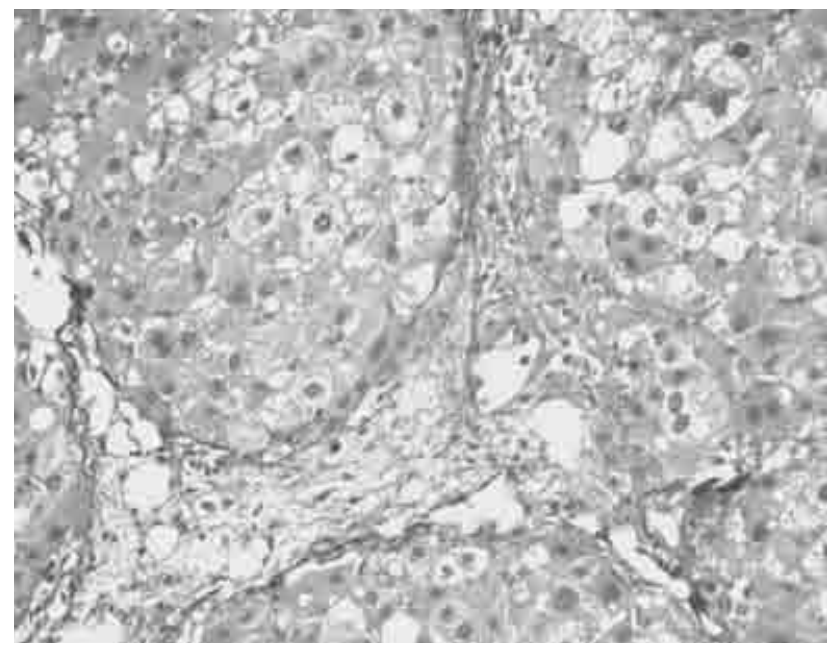

Fig. 1d: Cirrhotic liver, van Gieson technique.

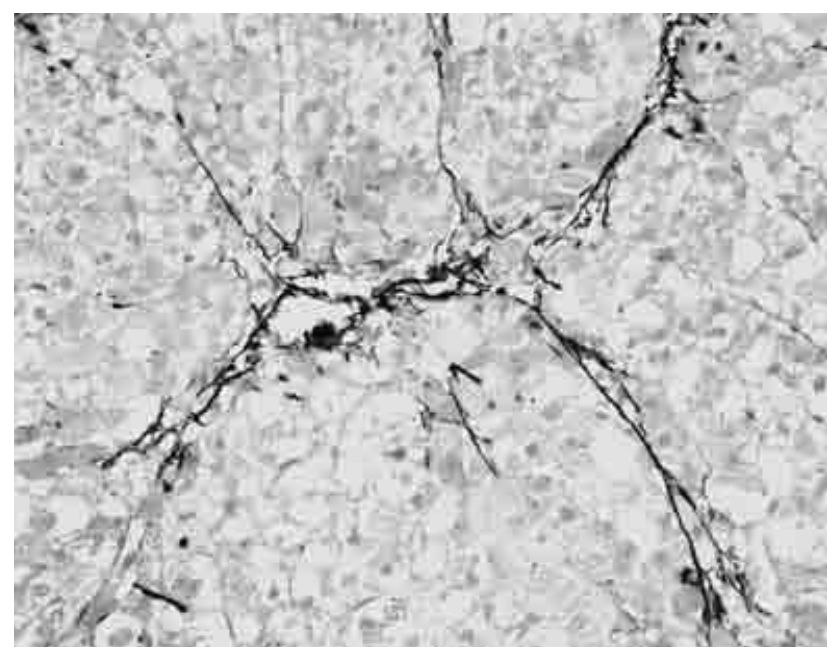

Fig. 1f: Cirrhotic liver, Gömöri's silver impregnation. 


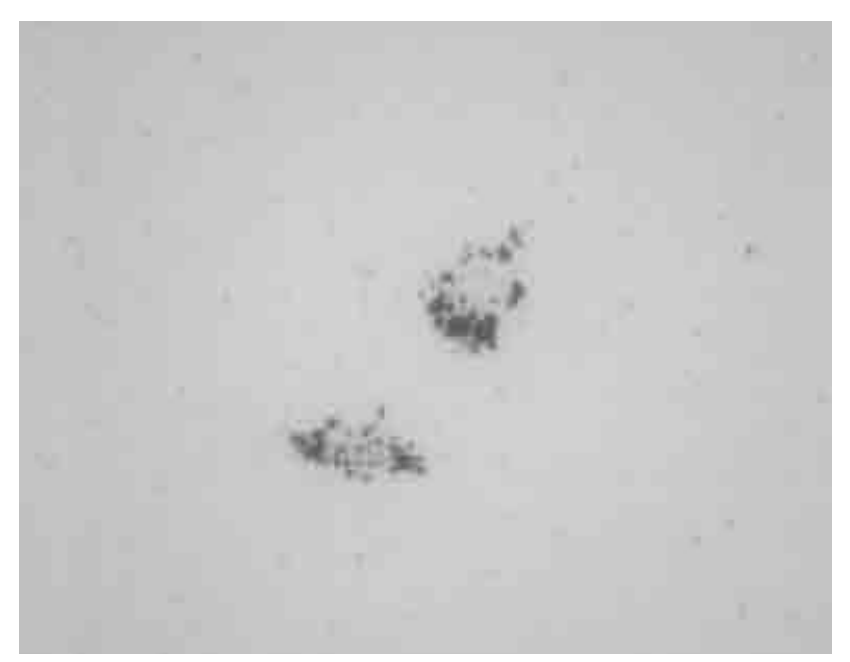

Fig. 2a: HSC from normal liver, 2 days in culture, Oil Red O.

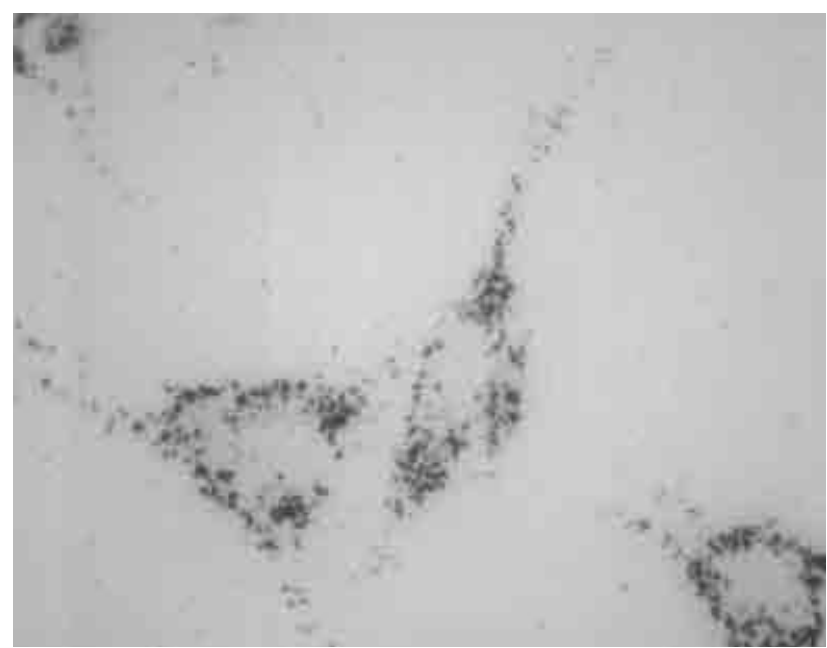

Fig. 2c: HSC from cirrhotic liver, 2 days in culture, Oil Red O.

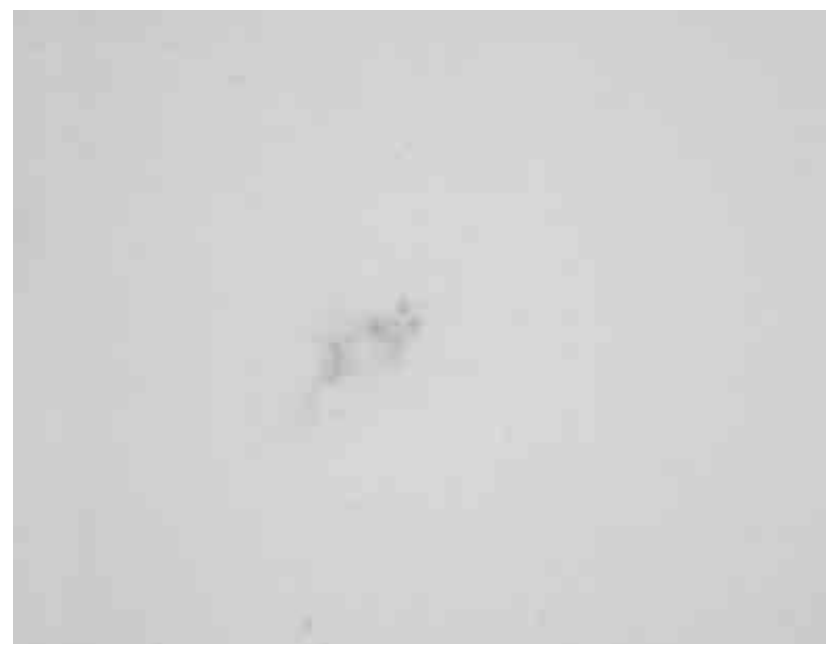

Fig. 3a: HSC from normal liver, 2 days in culture, $\alpha$-SMA.

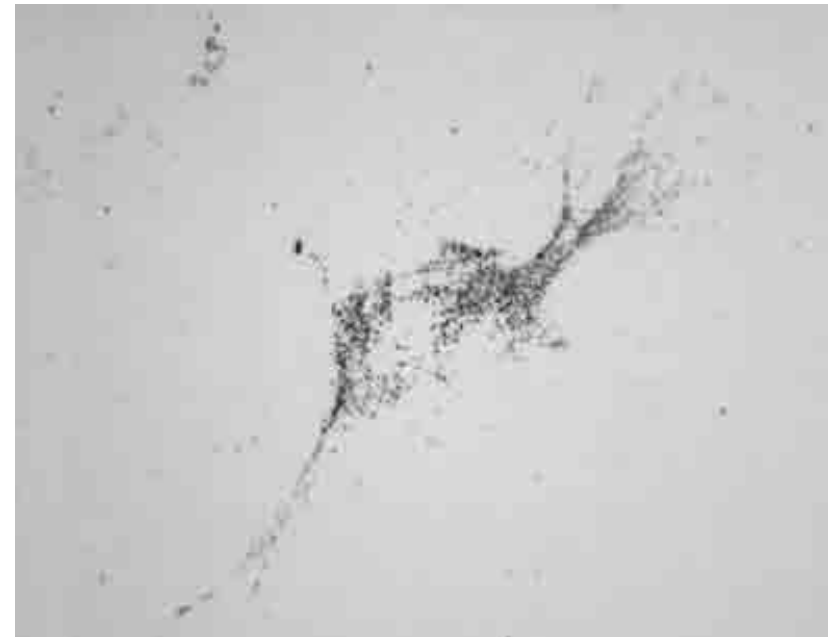

Fig. 2b: HSC from normal liver, 7 days in culture, Oil Red.

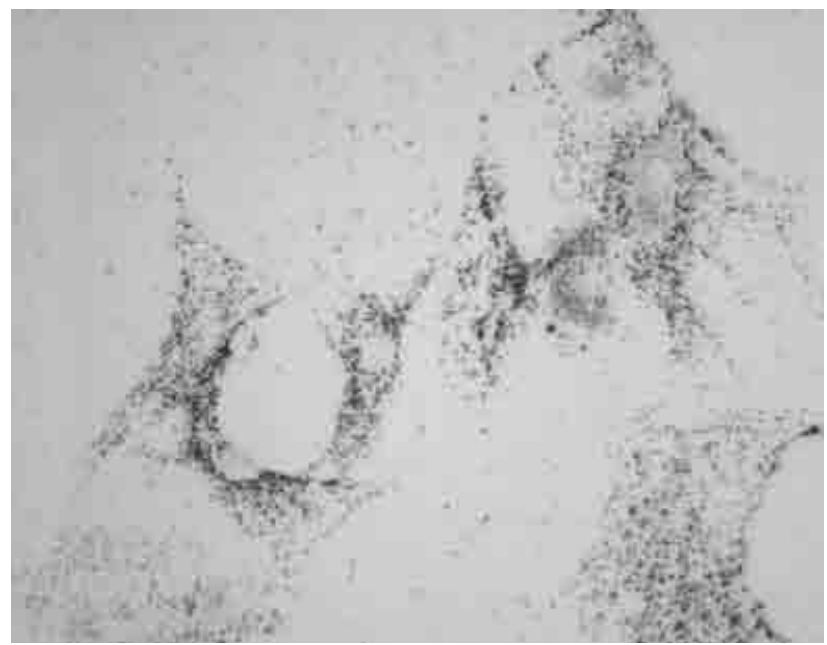

Fig. 2d: HSC from cirrhotic liver, 7 days in culture, Oil Red O.

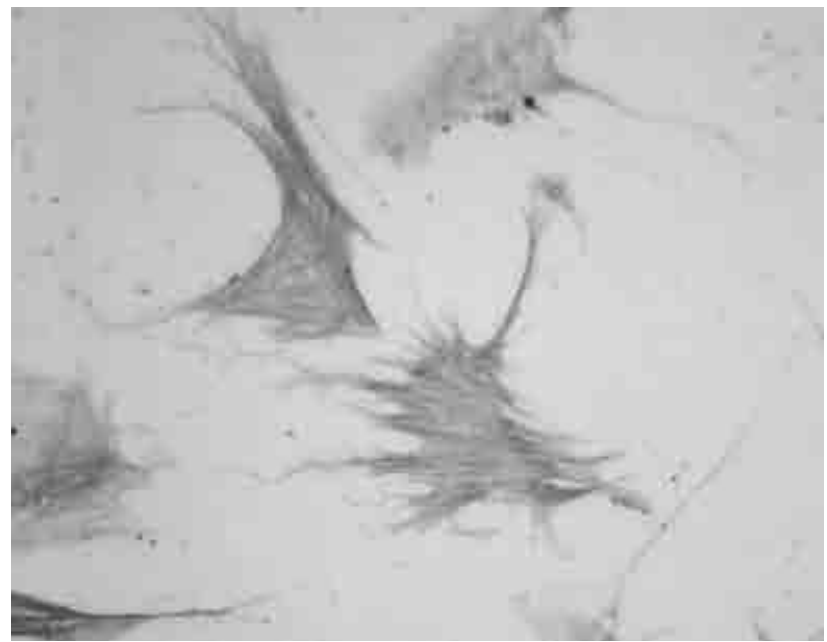

Fig. 3b: HSC from normal liver, 7 days in culture, $\alpha$-SMA. 


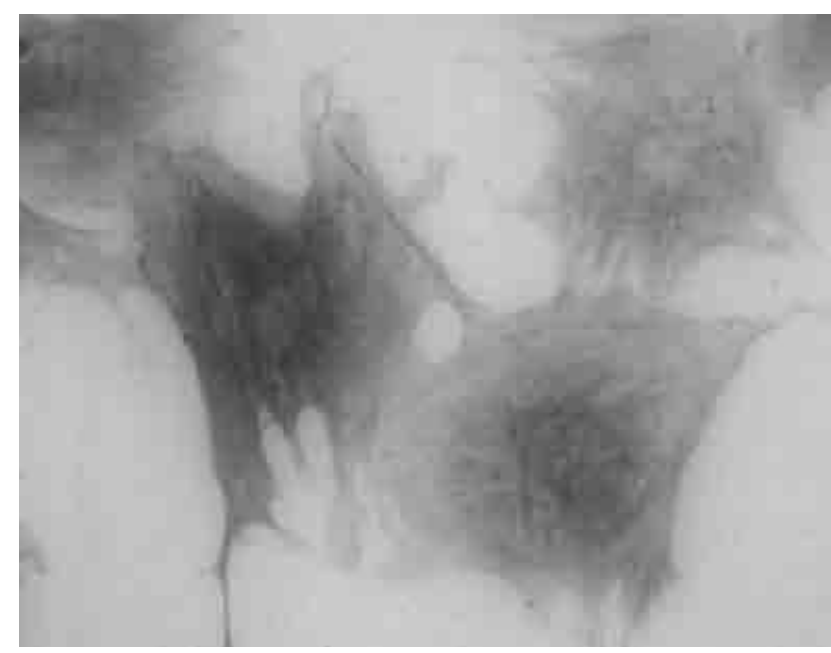

Fig. 3c: HSC from cirrhotic liver, 2 days in culture, $\alpha$-SMA.

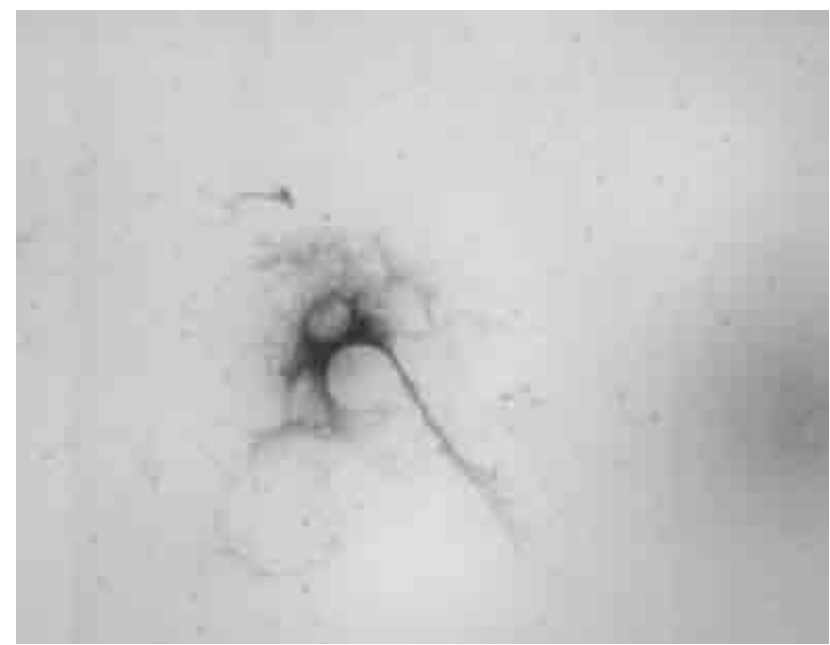

Fig. 4a: HSC from normal liver, 2 days in culture, vimentin.

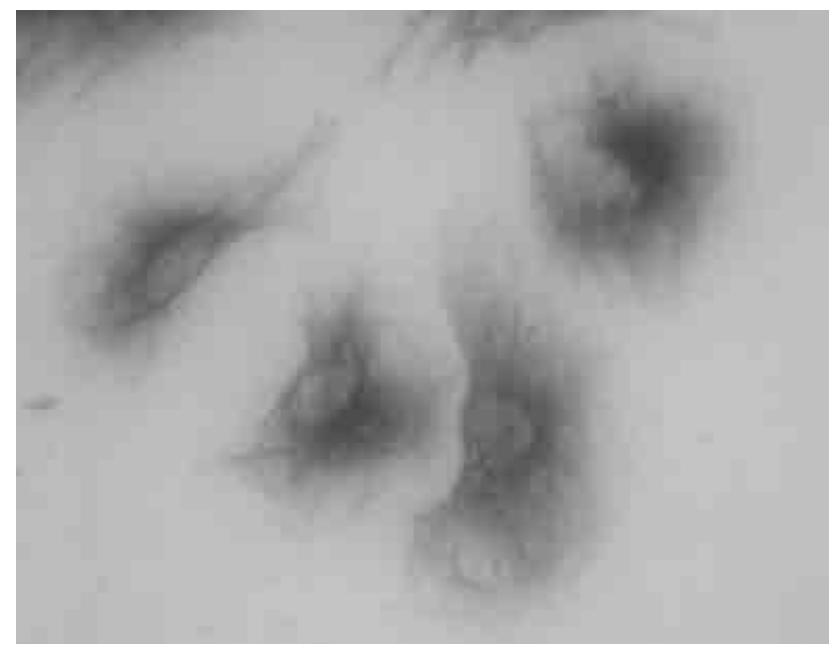

Fig. 4c: HSC from cirrhotic liver, 2 days in culture, vimentin.

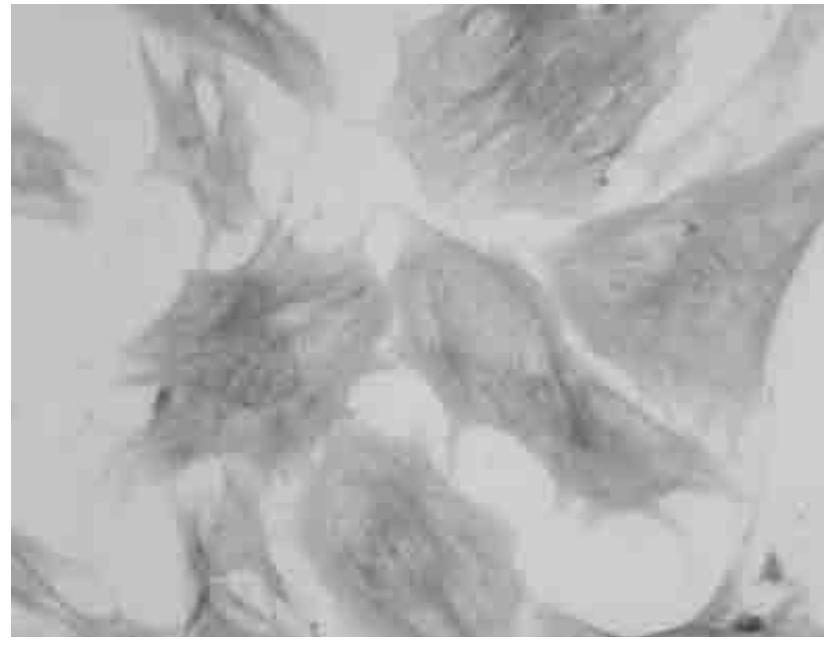

Fig. 3d: HSC from cirrhotic liver, 7 days in culture, $\alpha-S M A$.

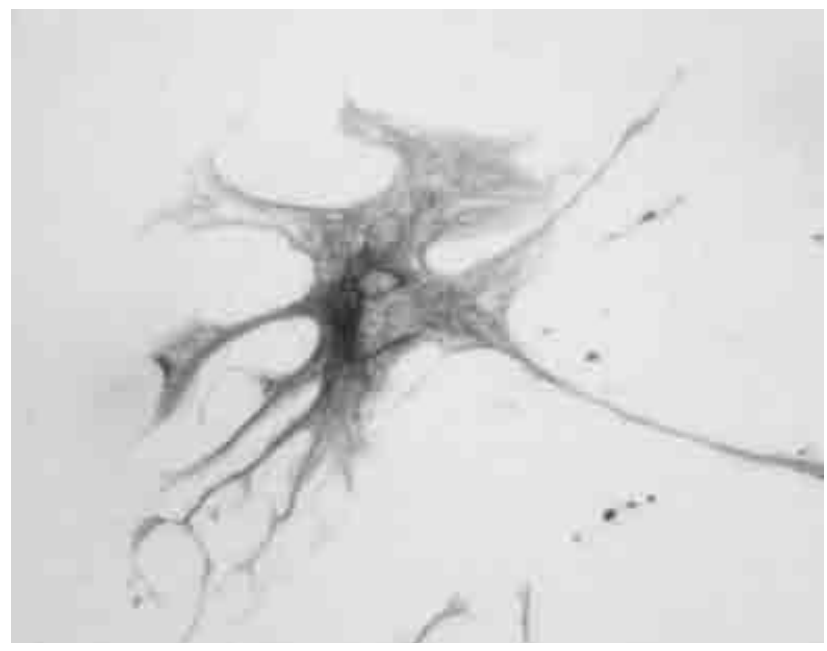

Fig. 4b: HSC from normal liver, 7 days in culture, vimentin.

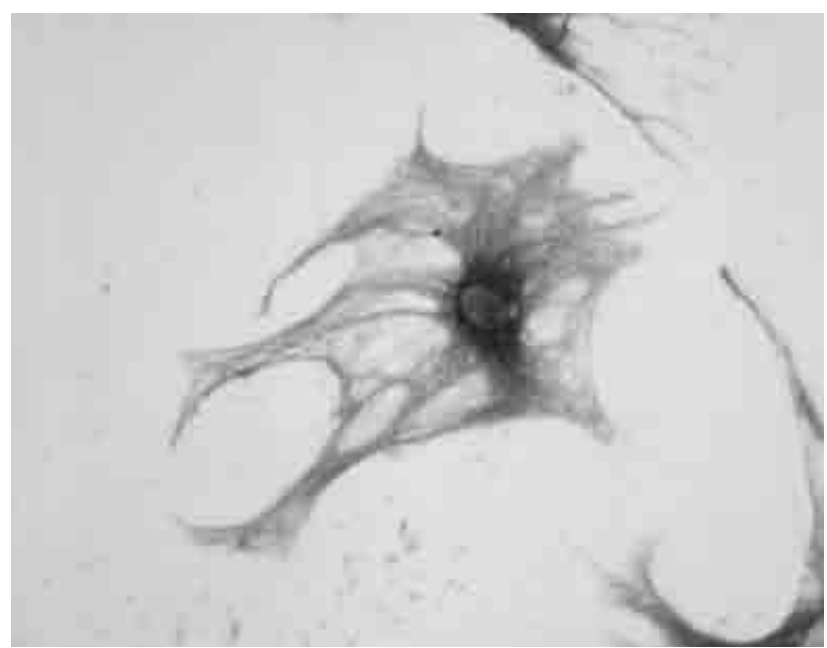

Fig. 4d: HSC from cirrhotic liver, 7 days in culture, vimentin. 


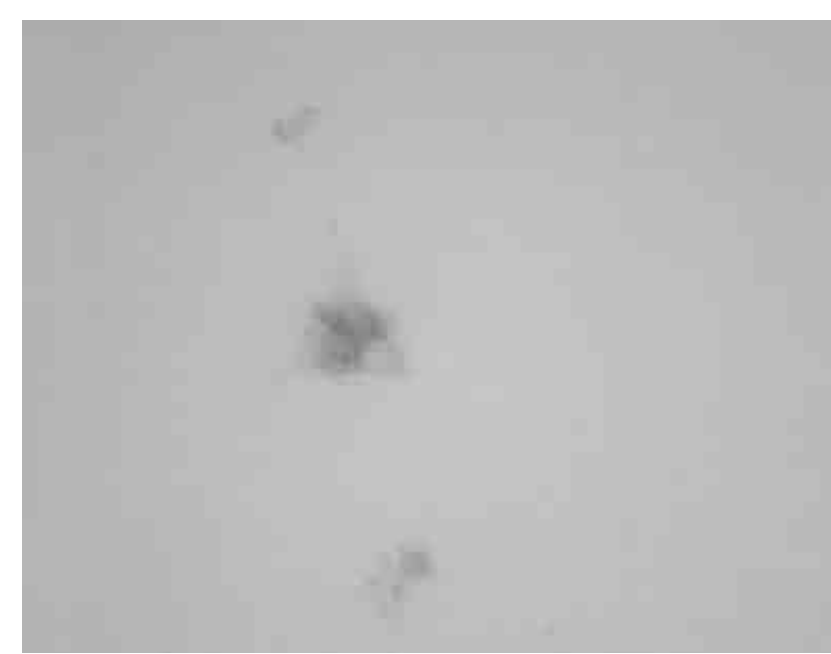

Fig. 5a: HSC from normal liver, 2 days in culture, desmin.

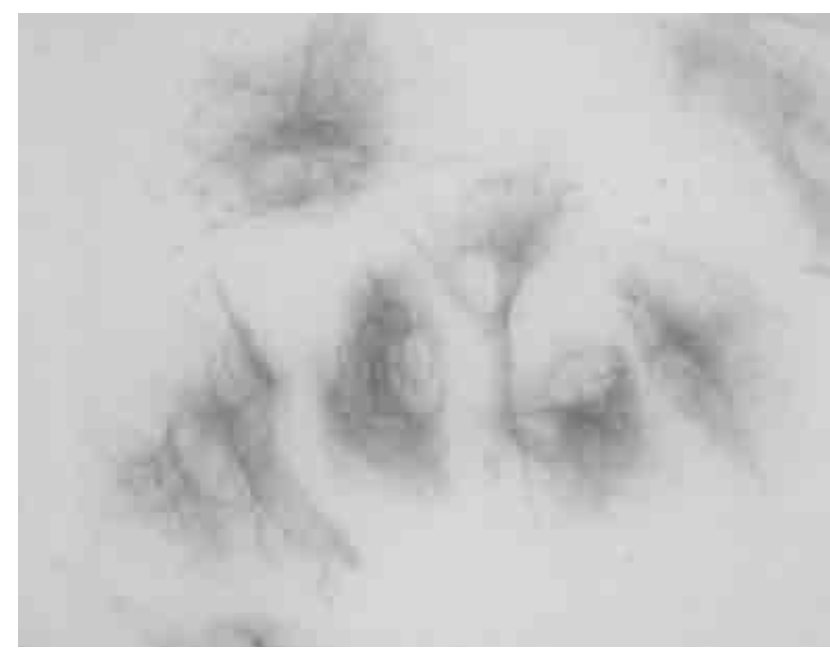

Fig. 5c: HSC from cirrhotic liver, 2 days in culture, desmin.

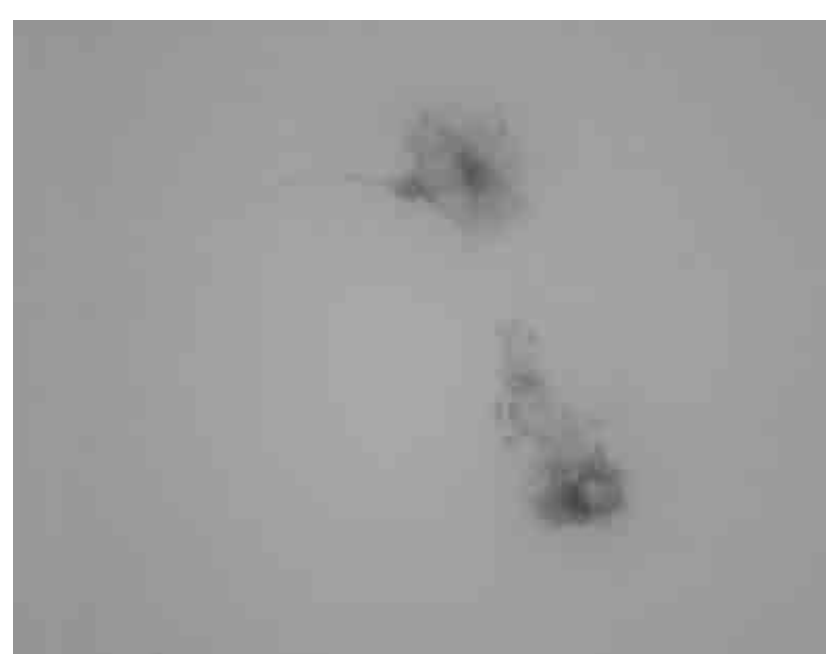

Fig. 6a: HSC from normal liver, 2 days in culture, GFAP.

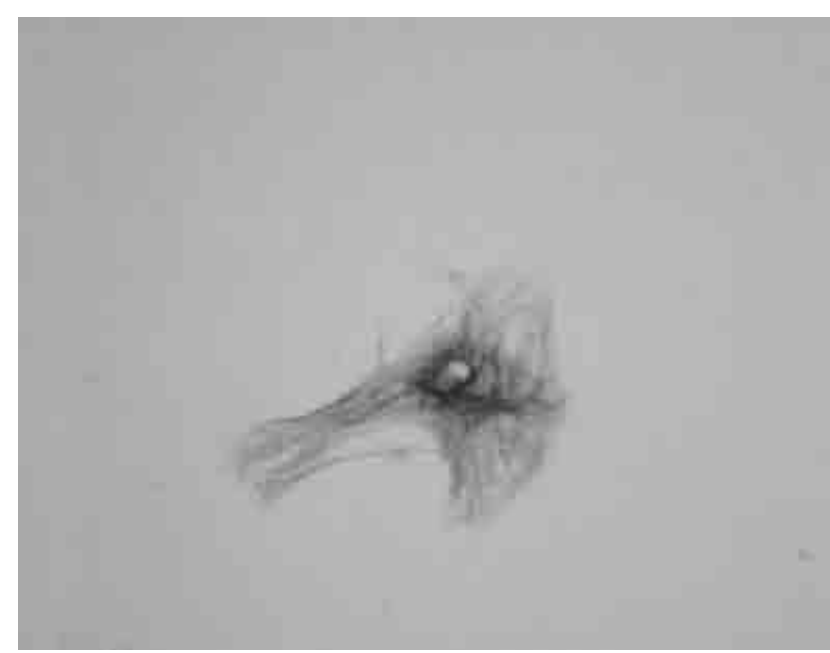

Fig. 5b: HSC from normal liver, 7 days in culture, desmin.

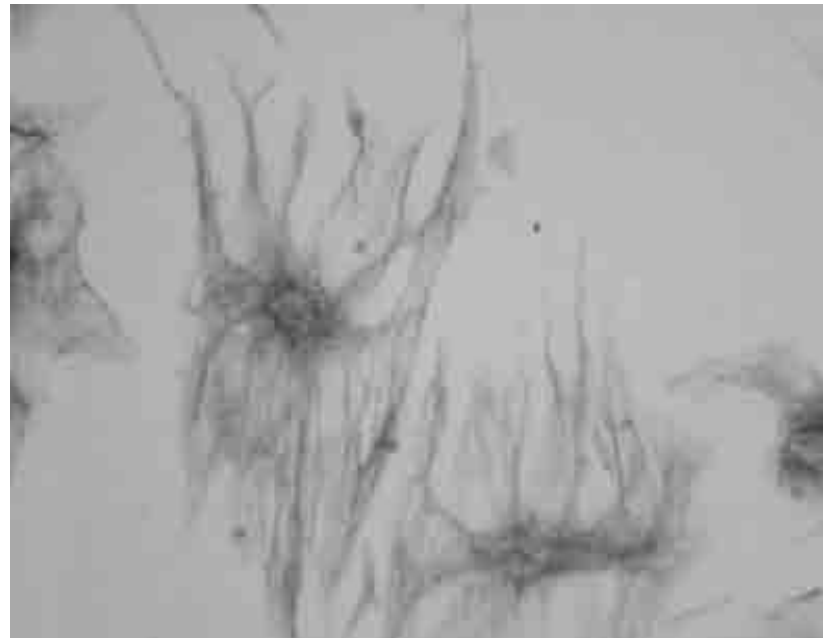

Fig. 5d: HSC from cirrhotic liver, 7 days in culture, desmin.

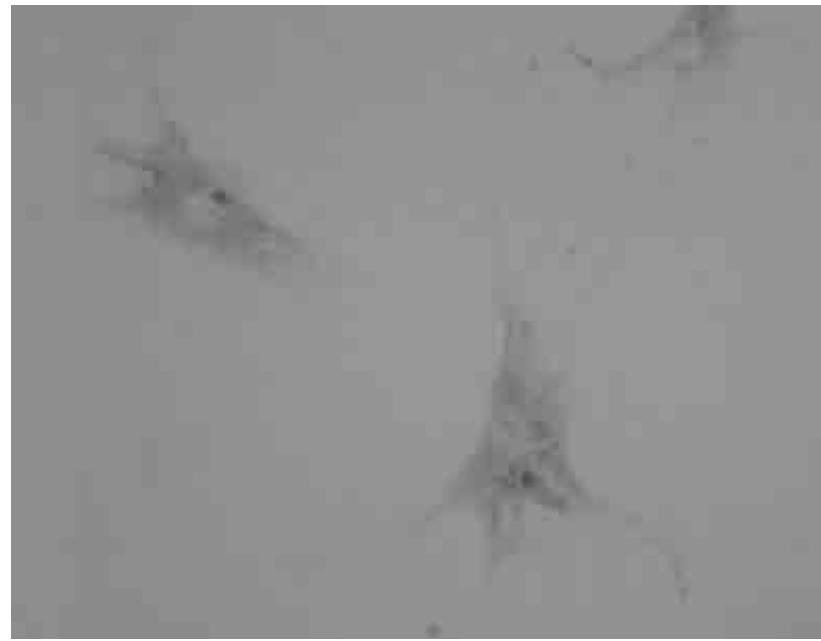

Fig. 6b: HSC from normal liver, 7 days in culture, GFAP. 


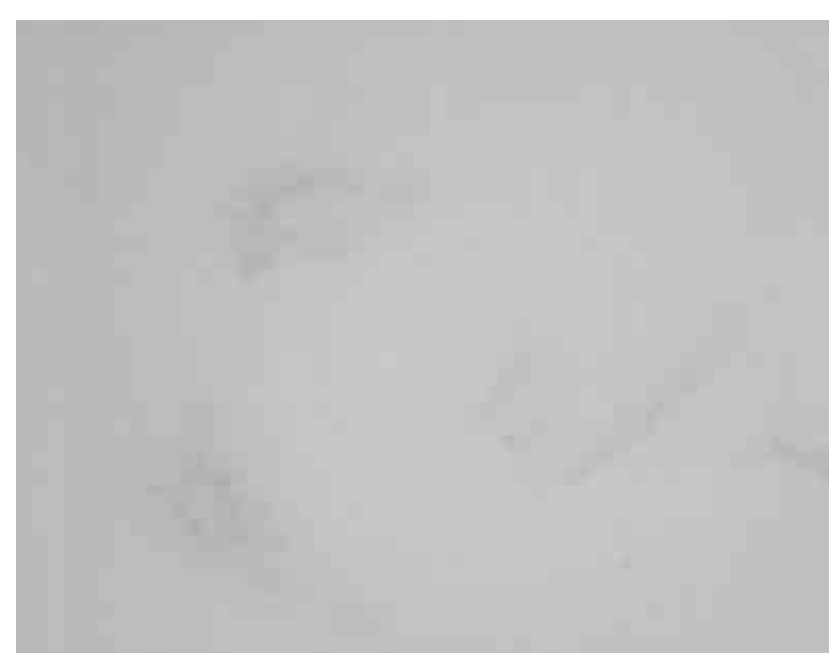

Fig. 6c: HSC from cirrhotic liver, 2 days in culture, GFAP.

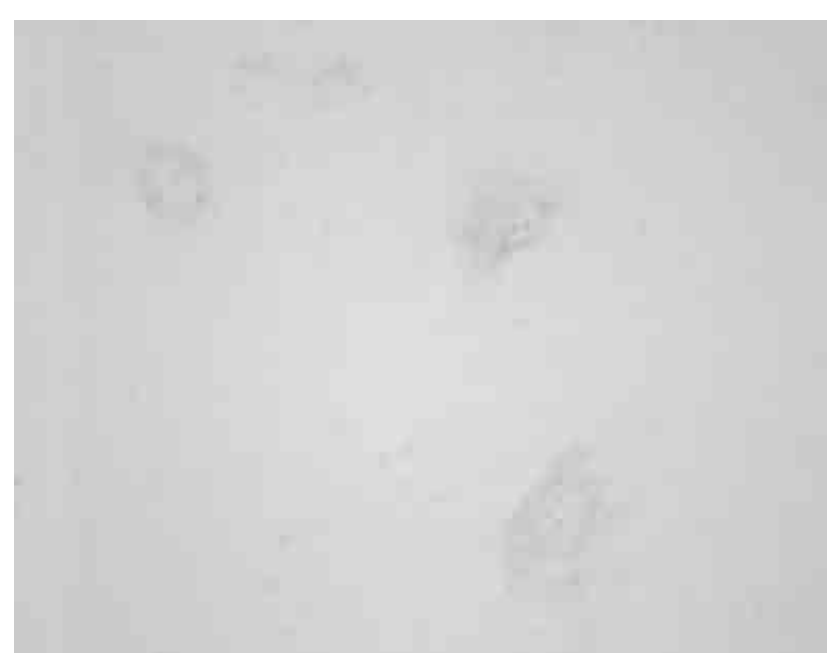

Fig. 6d: HSC from cirrhotic liver, 7 days in culture, GFAP.

Tab. 1: The expression of cytoskeletal antigens in HSC from normal and cirrhotic liver.

\begin{tabular}{|c|c|c|c|c|}
\hline $\begin{array}{c}\text { Days in } \\
\text { culture }\end{array}$ & 2 & 2 & $\mathrm{~N}$ & $\mathrm{C}$ \\
\hline$\alpha$-SMA & - & $\begin{array}{c}++ \\
80 \%\end{array}$ & $\begin{array}{c}++ \\
50 \%\end{array}$ & $\begin{array}{c}+++ \\
100 \%\end{array}$ \\
\hline Vimentin & $\begin{array}{c}++ \\
100 \%\end{array}$ & $\begin{array}{c}+++ \\
100 \%\end{array}$ & $\begin{array}{c}+++ \\
100 \%\end{array}$ & $\begin{array}{c}+++ \\
100 \%\end{array}$ \\
\hline Desmin & $\begin{array}{c}+ \\
45-60 \%\end{array}$ & $\begin{array}{c}++ \\
60 \%\end{array}$ & $\begin{array}{c}++ \\
45-60 \%\end{array}$ & $\begin{array}{c}+++ \\
95-100 \%\end{array}$ \\
\hline GFAP & $\begin{array}{c}++ \\
100 \%\end{array}$ & - & - & - \\
\hline
\end{tabular}

The intensity of staining is indicated (- no staining,,,++++++ increasing intensity).

$\mathrm{N}$ - normal liver, $\mathrm{C}$ - cirrhotic liver. The percentage of positive cells was determined by counting HSC in the cultures originating from 3 normal and 3 cirrhotic liver. Three hundred cells were counted in each culture and the range of the results is shown.

was poorly expressed in normal cells 2 days after isolation but its expression increased in culture. About $50 \%$ cells were positive and this number did not change during cultivation. In contrast, desmin expression in the cells from cirrhotic liver was prominent in more than a half of cells at 2 days and the number of positive cells increased to $95-100 \%$ at 7 days (Fig. 5 a-d). The expression of GFAP was found in normal cells at 2 days when all cells were labelled. The marker was lost during cultivation. The HSC from cirrhotic livers were negative at both time intervals (Fig. $6 \mathrm{a}-\mathrm{d}$ ). The results of antigen analysis are summarized in Table 1 .

\section{Discussion}

Carbon tetrachloride-induced cirrhosis in rats resembles human disease in some features: extensive necrosis, nodules of regenerating hepatocytes surrounded by connective tissue septa and a severalfold increase in collagen content (14). Nine-week treatment of rats with carbon tetrachloride with 2 intragastric doses of the toxin per week results in fairly reproducible fully developed liver cirrhosis (1).

Quiescent HSC found in normal liver do not express $\alpha$-SMA, the marker of HSC activation. Cytoplasmic immunoreactivity against this antigen appears when isolated HSC grow on plastic substratum and spontaneously transform to myofibroblast-like cells $(11,18)$. In agreement with this theory, the HSC from normal liver were negative for $\alpha$-SMA early after isolation. Judging by their $\alpha$-SMA content, a majority of HSC obtained from cirrhotic liver were already activated 2 days after isolation and after 7 days of culture the number of activated, strongly positive cells reached $100 \%$

Vimentin, desmin and GFAP are intermediate filament proteins. Vimentin is consistently found in normal HSC 
and other mesenchymal cells $(10,18)$. It was also strongly expressed in the HSC we isolated from the cirrhotic liver.

Desmin is a protein necessary for proper functioning of muscle (13). It is also found in HSC of rat origin and suggests their relationship to muscle cells. Only a part of normal HSC express this antigen (11) which was also reflected in our results. However, almost all HSC from cirrhotic liver were strogly positive for desmin. It is still an open question if there is a functional difference between desmin-positive and desmin-negative HSC (10).

GFAP is a glial-specific protein found in long processes of astrocytes (20). It was suggested as a specific marker for HSC (12). It seems to be expressed in quiescent cells only. It disappeared in normal cells after a week of culture and was not found in HSC from cirrhotic liver.

The loss of lipid droplets is considered by some authors a sign of HSC transdifferentiation into myofibroblast-like cells $(5,15)$. We did not observe any significant change in Oil Red staining in the course of HSC activation.

As an introduction to our studies of gene expression in hepatic stellate cells in liver cirrhosis we used four cytoskeletal antigens to characterize these cells isolated from cirrhotic liver and to compare them with HSC isolated from normal rat liver. Using immunocytochemical methods we have found that the cells from cirrhotic liver show similar tendencies in cytoskeletal antigen expression as normal HSC activated on plastic but the changes are more profound. Both the intensity of antigen expression and the number of cells involved are usually larger in cirrhotic cells than in normal ones.

\section{Acknowledgment}

This study was supported by grants GAČR 305/03/ 1513, MSM 002160820 and MSM 111500003

\section{References}

1. Bickel M, Baringhaus KH, Gerl M, Günzler V, Kanta J, Schmidts L, Stapf M, Tschank G, Weidman K, Werner U. Selective inihibition of hepatic collagen accumulation in experimental liver fibrosis in rats by a new prolyl 4-hydroxylase inhibitor. Hepatology 1998;28:404-411

2. Buniatian G, Hamprecht B, Gebhardt R. Glial fibrillary acidic protein as a marke of perisinusoidal stellate cells that can distinguish between the normal and myofibroblast-like phenotypes. Biol Cell 1996;87:65-73

3. Elsharkawy AM, Wright MC, Hay RT, Arthur MJP, Hughes T, Bahr MJ, Degitz $\mathrm{K}$, Mann DA. Persistent activation of nuclear factor- $\mathrm{\kappa B}$ in cultured rat hepatic stellate cells involves the induction of potentially novel Rel-like factors and pro- longed changes in the expression of IKB family proteins. Hepatology 1999; 30:761-769

4. Friedman SL, Roll FJ, Bozles J, Arenson DM, Bissell DM. Maintenance of differentiated phenotype of cultured rat hepatic lipocytes by basement membrane matrix. J Biol Chem 1989;264:10756-10762

5. Friedman SL. The cellular basis of hepatic fibrosis. Mechanisms and treatment strategies. New England J Med 1993; 328:1828-1835

6. Geerts A, Vrijsen R, Rauterberg J, Burt A, Schellinck, Wisse E. In vitro differentiation of fat-storing cells parallels marked increase of collagen synthesis and secretion. J Hepatol 1989;9:59-68

7. Geerts A, Niki T, Hellemans K, De Craemer D, Van Den Berg K, Lazou JM, Stange G, Van De Winkel, De Bleser P. Purification of rat hepatic stellate cells by side scatter-activated cell sorting. Hepatology 1998;27:590-598

8. Gressner AM. The cell biology of liver fibrogenesis - an imbalance of proliferation, growth arrest and apoptosis of myofibroblasts. Cell Tissue Res 1998; 292:447-452

9. Knook DL, Sefelaar AM, de Leeuw. Fat-storing cells of the rat liver. Exp Cell Res 1982;139:468-472

10. Li D, Friedman SL. Hepatic stellate cells: morphology, function, and regulation In: Arias IM, Boyer JL, Chisari FV, Fausto N, Schachter D, Shafritz DA, eds. The Liver: Biology and Pathobiology. Philadelphia: Lippincott Williams and Wilkins, 2001: 455-468

11. Mathew J, Geerts A, Burt AD. Pathobiology of hepatic stellate cells. HepatoGastroenterol 1996;43:72-91

12. Neubauer K, Knittel T, Aurisch S, Fellmer P, Ramadori G.. Glial fibrillary acidic protein - a cell type specific marker for Ito cells in vivo and in vitro. J Hepatol 1996; 24:719-730

13. Paulin D, Li Z. Desmin: a major intermediate filament protein essential for the structural integrity and function of muscle. Exp Cell Res 2004; 301:1-7

14. Pérez Tamayo $\mathrm{R}$. Is cirrhosis of the liver experimentally produced by $\mathrm{CCl}_{4}$ an adequate model of human cirrhosis? Hepatology $1983 ; 3: 112-120$

15. Pinzani M. Novel insights into the biology and physiology of the Ito cell Pharmac Ther 1995; 66:387-412

16. Pinzani M, Gentilini P. Biology of hepatic stellate cells and their possible relevance in the pathogenesis of portal hypertension in cirrhosis. Semin Liver Dis 1999;19:397-410

17. Ramadori G, Veit T, Schwögler S, Dienes HP, Knittel T, Rieder H, Meyer zum Büschenfelde KH. Virchows Arch B Cell Pathol 1990;59:349-357

18. Rockey DC, Friedman SL. Cytoskeleton of liver perisinusoidal cells (lipocytes) in normal and pathological conditions. Cell Motil Cytoskeleton 1992;22:227-234

19. Senoo H. Structure and function of hepatic stellate cells. Med Electron Microsc 2004;37:3-15

20. Weinstein DE, Shelanski ML, Liem RKH. Suppression by antisense mRNA demonstrates a requirement for the glial fibrillary acidic protein in the formation of stable astrocytic processes in response to neurons. J Cell Biol 1991; 112:1205-1213

Submitted May 2005.

Accepted July 2005.

Doc. RNDr. Jiři Kanta, CSc., Charles University in Prague, Faculty of Medicine in Hradec Králové, Department of Medical Biochemistry, Šimkova 870, 50038 Hradec Králové, Czech Republic. e-mail: kanta@lfhk.cuni.cz 